adipokine adiponectin and increased levels of proinflammatory proteins fibrinogen and C-reactive protein (CRP, detected by highsensitivity assay). Weight loss should improve levels of these markers, but study findings are conflicting. Madsen and colleagues investigated the effect of weight loss on levels of adiponectin, fibrinogen and CRP in a SMOMS substudy (a randomized, controlled trial of the lipase inhibitor orlistat).

Participants were 93 obese individuals (mean BMI $37 \mathrm{~kg} / \mathrm{m}^{2}$; mean age 45.8 years) who completed 8 weeks of a very-low-energy diet (VLED) followed by 3 years of lifestyle intervention and either placebo or orlistat treatment. Serum levels of CRP and adiponectin (in 68 participants), and fibrinogen (in 93 participants) were measured at baseline, and 8 weeks, 6, 12 and 36 months after the VLED ceased. Mean weight loss was $14.3 \mathrm{~kg}$ after the VLED, and $7.7 \mathrm{~kg}$ after 3 years because some weight regain occurred. Changes in marker levels were not significantly different between groups; therefore, groups were combined for the marker-level analysis. After weight loss, adiponectin levels increased and CRP and fibrinogen levels decreased; however, a net weight loss $>10 \%$ seemed to be required for these improvements to be sustained.

The finding that substantial weight loss is required to improve levels of the three markers could explain why previous studies reported conflicting findings.

Original article Madsen EL et al. (2008) Weight loss larger than $10 \%$ is needed for general improvement of levels of circulating adiponectin and markers of inflammation in obese subjects: a 3-year weight loss study. Eur J Endocrinol 158: $179-187$

\section{Insulin resistance in offspring of mothers with gestational diabetes}

Maternal hyperglycemia is associated with insulin resistance in offspring in high-risk (e.g. Pima Indian) populations, but this association remains unconfirmed in low-risk populations. Keely and colleagues' previous randomized, controlled trial in 299 women (91\% white) with gestational diabetes mellitus compared the effects of minimal intervention or tight glycemic control, respectively, on perinatal outcomes. These researchers have now investigated the prevalence of insulin resistance in offspring (aged 7-11 years) of those women.

In total, 68 children underwent a $2 \mathrm{~h}$ oral glucose tolerance test, and their $8 \mathrm{~h}$ fasting levels of plasma glucose, insulin, HDL cholesterol, total cholesterol and triglycerides, as well as waist circumference and BMI were measured. Insulin resistance was quantified using the homeostasis model assessment (HOMA). At least one HOMA marker of insulin resistance was present in 23 children; however, 2 h or fasting plasma glucose abnormalities were rare. Hypertriglyceridemia was the most common abnormality, present in $21 \%$ of the children. After adjustment for potential confounding variables, waist circumference and triglyceride level were significant predictors of insulin resistance. Notably, maternal and offspring triglyceride and HDL cholesterol levels, waist circumference and HOMA insulin-resistance markers were significantly correlated, but $2 \mathrm{~h}$ or fasting plasma glucose levels were not.

Children of mothers with gestational diabetes mellitus seem to have an increased risk of insulin resistance and the metabolic syndrome, but whether this risk is influenced by control of maternal glycemia remains unclear. Screening strategies based on blood-glucose levels alone might be inadequate to identify these children.

Original article Keely EJ et al. (2008) Prevalence of metabolic markers of insulin resistance in offspring of gestational diabetes pregnancies. Pediatr Diabetes 9: 53-59

\section{rhGH therapy does not unmask central hypoadrenalism in children with idiopathic GHD}

Adults with organic growth-hormone deficiency (GHD) treated with recombinant human growth hormone $(\mathrm{rhGH})$ require reassessment of their hypothalamic-pituitary-adrenal (HPA) axis function because this therapy could unmask central hypoadrenalism. Whether children with idiopathic GHD require HPA reassessment during rhGH therapy is, however, unknown. Giavoli et al. evaluated HPA function in children with idiopathic GHD before and during rhGH therapy.

This single-center study included 10 consecutive children (mean age 12.2 years, 5 boys) with idiopathic, isolated GHD. Before rhGH treatment, all children had normal HPA function and pituitary MRI findings. At baseline and 